\title{
A Simple Multicloud Parameterization for Convectively Coupled Tropical Waves. Part I: Linear Analysis
}

\author{
BOUALEM KHOUIDER \\ Department of Mathematics and Statistics, University of Victoria, Victoria, British Columbia, Canada \\ ANDReW J. MAJDA \\ Department of Mathematics, and Center for Atmosphere/Ocean Sciences, Courant Institute, New York University, \\ New York, New York
}

(Manuscript received 13 May 2005, in final form 14 September 2005)

\begin{abstract}
Recent observational analysis reveals the central role of three multicloud types, congestus, stratiform, and deep convective cumulus clouds, in the dynamics of large-scale convectively coupled Kelvin waves, westward-propagating two-day waves, and the Madden-Julian oscillation. A systematic model convective parameterization highlighting the dynamic role of the three cloud types is developed here through two baroclinic modes of vertical structure: a deep convective heating mode and a second mode with low-level heating and cooling corresponding respectively to congestus and stratiform clouds. A systematic moisture equation is developed where the lower troposphere moisture increases through detrainment of shallow cumulus clouds, evaporation of stratiform rain, and moisture convergence and decreases through deep convective precipitation. A nonlinear switch is developed that favors either deep or congestus convection depending on the relative dryness of the troposphere; in particular, a dry troposphere with large convective available potential energy (CAPE) has no deep convection and only congestus clouds. The properties of the multicloud model parameterization are tested by linearized analysis in a two-dimensional setup with no rotation with constant sea surface temperature. In particular, the present study reveals new mechanisms for the large-scale instability of moist gravity waves with features resembling observed convectively coupled Kelvin waves in realistic parameter regimes without any effect of wind-induced surface heat exchange (WISHE). A detailed dynamical analysis for the linear waves is given herein and idealized nonlinear numerical simulations are reported in a companion paper. A maximum congestus heating leads during the dry phase of the wave. It is followed by an increase of the boundary layer $\theta_{e}$, that is, CAPE, and lower troposphere moistening that precondition the upper troposphere for the next deep convective episode. In turn, deep convection consumes CAPE and removes moisture, thus yielding the dry episode.
\end{abstract}

\section{Introduction}

The chicken-egg problem of the mutual interaction between tropical large-scale circulation and moist convection has been an area of active research during the last few decades. Moreover, contemporary general circulation models (GCMs) often perform poorly in parameterizing and/or resolving the observed large-scale features of organized tropical convection, such as convectively coupled waves, as well as their impact on planetary-scale tropical circulation (Slingo et al. 1996; Mon-

Corresponding author address: Dr. Boualem Khouider, Dept. of Mathematics and Statistics, University of Victoria, P.O. Box 3045 STN CSC, Victoria, BC V8W 3P4, Canada.

E-mail: khouider@math.uvic.ca crieff and Klinker 1997); the reasons for such poor performance are not well understood. Intermediate models with crude vertical resolution, typically involving a single baroclinic vertical mode, have been used for theoretical and numerical studies of various strategies for parameterizing moist convection and convectively coupled waves (Emanuel 1987; Mapes 1993; Neelin and Yu 1994; Yano et al. 1995; Yano et al. 1998; Majda and Shefter 2001a; Majda and Khouider 2002; Frierson et al. 2004). Although a lot of progress has been made in the understanding of this meteorological phenomenon, the problem remains open, practically important, and indeed challenging.

Two types of models have dominated the arena of tropical moist convection: convergence-driven models and quasi-equilibrium models. Convergence models 
date back to the work of Charney and Eliassen (1964), followed by Yamasaki (1969), Hayashi (1971), and Lindzen (1974). The convergence models, also called convective instability of second kind (CISK) models, sustain convection through large reservoirs of convective available potential energy (CAPE) driven by lowlevel convergence. Such models exhibit extreme sensitivity to grid-scale behavior and linearized stability analysis reveals the undesirable feature of catastrophic instability with increasingly larger growth rates on the smallest scales (Yano et al. 1998; Majda and Shefter 2001a). In the quasi-equilibrium thinking, first introduced by Arakawa and Shubert (1974), one assumes a large-scale quasi-equilibrium state where CAPE is nearly constant and deep convection acts as an energy regulator in restoring quickly the equilibrium by consuming any excess of CAPE. The triggering and the amplification of convection in quasi-equilibrium models rely on surface fluxes. Indeed, such quasi-equilibrium models are linearly (Neelin and $\mathrm{Yu}$ 1994) and even nonlinearly stable (Frierson et al. 2004). The most popular mechanism used in concert with the quasiequilibrium models to create instability is wind-induced surface heat exchange (WISHE; Emanuel 1987; Emanuel et al. 1994).

Recent analysis of observations over the warm pool in the Tropics reveals the ubiquity of three cloud types above the boundary layer: shallow congestus clouds, stratiform clouds, and deep penetrative cumulus clouds (Lin and Johnson 1996; Johnson et al. 1999). Furthermore, recent analysis of convectively coupled waves on the large scales reveals a similar multicloud convective structure with leading shallow congestus cloud decks that moisten and precondition the lower troposphere followed by deep convection and finally trailing decks of stratiform precipitation; this structure applies to the eastward propagating convectively coupled Kelvin waves (Wheeler and Kiladis 1999; Straub and Kiladis 2002) and westward-propagating two-day waves (Haertl and Kiladis 2004), which reside on equatorial synoptic scales of order 1000 to $3000 \mathrm{~km}$ in the lower troposphere as well as the planetary-scale MaddenJulian oscillation (Kiladis et al. 2005; Dunkerton and Crum 1995). An inherently multiscale theory for the Madden-Julian oscillation with qualitative agreement with observations that is based on these three cloud types has been developed recently (Majda and Biello 2004; Biello and Majda 2005). While there is no doubt that WISHE plays an important role in hurricane development (Zehnder 2001; Craig and Gray 1996), there is no observational evidence directly linking the structure of convectively coupled Kelvin waves and two-day waves to WISHE.
Furthermore, despite the observational evidence, none of the models with a single vertical mode mentioned earlier account for the multimode nature of tropical convection and the importance of the different cloud types; shallow/congestus, stratiform and deeppenetrative cumulus clouds. They are concentrated solely on the deep-penetrative and shallow boundary layer clouds. Parameterizations with two convective heating modes systematically representing, a deepconvective mode and a stratiform mode, have first appeared in the work of Mapes (2000). Majda and Shefter (2001b, hereafter MS01) proposed a much simpler systematic version of Mapes' model based on a Galerkin projection of the primitive equations onto the first two linear baroclinic modes yielding a set of two shallowwater systems. The first baroclinic system is heated by the deep convective clouds while the second baroclinic system is heated aloft by the stratiform clouds. Linear stability analysis of this model convective parameterization revealed a mechanism of stratiform instability independent of WISHE (MS01; Majda et al. 2004, hereafter M04). Direct numerical simulations carried out in M04 revealed the resemblance of many features of the moist gravity waves for the MS01 model and the real world convective superclusters as depicted in M04 and in observational papers (e.g., Straub and Kiladis 2002). One visible shortcoming of the MS01 model is however its short-cutting of the role of the shallow/congestus heating as in the early Mapes' model. Also, inherited from the quasi-equilibrium school (Yano and Emanuel 1991; Yano et al. 1998), the MS01 model uses very sensitive parameters that are nonphysically kept fixed/ constant and spatially homogeneous, such as the precipitation efficiency and the area fraction of deep convection.

In the present paper, we propose a new model convective parameterization, within a framework similar to the MS01 model. In addition to the deep convective and stratiform clouds, the present model carries cumulus congestus clouds, which serve to heat the second baroclinic mode from below and cool it from above as in actual congestus cloud decks. The new model is based on a self-consistent derivation and it avoids many of the commonly used ad hoc parameters. We systematically derive an equation for the vertically integrated water vapor with mean vertical background moisture profile forced by both the first baroclinic and second baroclinic (low level) convergence within the physical constraints of conservation of vertically integrated moist static energy. Also the new model takes into account the dryness and moistness of the middle troposphere through a varying inhomogeneous switch parameter, $\Lambda$, in order to shut off or favor deep convec- 
tion and to increase or decrease the downdrafts from the cooling associated with evaporation of shallow clouds and stratiform rain. Moreover, the congestus convection is amplified whenever the middle troposphere is too dry to sustain deep convection and is shut off completely when deep convection is at its maximum. Furthermore, linear stability analysis reveals only large-scale instability in realistic parameter regimes and without any effect of WISHE. Thus, the new multicloud parameterizations are to some extent a marriage between the two schools of thought leading to the lowlevel convergence and quasi-equilibrium models.

The remainder of the paper is organized as follows. In section 2 we present the multicloud model parameterizations based on the two heating modes strategy. Radiative convective equilibrium solutions (RCEs) and the associated linearized equations are discussed in section 3. Section 4 presents the linear stability results and analysis for various parameter regimes. The physical structure and dynamical features of the associated unstable moist gravity waves are discussed in section 5 and a concluding discussion is given in section 6 . The details of the derivation of the vertical average moisture equation with first and second baroclinic convergence and the explicit linearized equations are given in appendices $\mathrm{A}$ and $\mathrm{B}$, respectively.

\section{The multicloud model}

\section{a. The dynamical core}

The dynamical core of the model convective parameterization proposed here consists of two coupled shallow-water systems corresponding to a direct heating/ deep convective mode and a second baroclinic-stratiform/congestus mode;

$$
\begin{aligned}
\frac{\partial \mathbf{v}_{j}}{\partial t}+\overline{\mathbf{U}} \cdot \nabla \mathbf{v}_{j}+\beta y \mathbf{v}_{j}^{\perp}-\nabla \theta_{j} & =-C_{d}\left(u_{0}\right) \mathbf{v}_{j}-\frac{1}{\tau_{R}} \mathbf{v}_{j} \\
\frac{\partial \theta_{1}}{\partial t}+\overline{\mathbf{U}} \cdot \nabla \theta_{1}-\operatorname{div} \mathbf{v}_{1} & =\frac{\pi}{2 \sqrt{2}} P+S_{1} \\
\frac{\partial \theta_{2}}{\partial t}+\overline{\mathbf{U}} \cdot \nabla \theta_{2}-\frac{1}{4} \operatorname{div} \mathbf{v}_{2} & =\frac{\pi}{2 \sqrt{2}}\left(-H_{s}+H_{c}\right)+S_{2} .
\end{aligned}
$$

In (2.1), $P \geq 0$ models the heating from deep convection while $H_{s}, H_{c}$ are the stratiform and congestus heating rates. As depicted in Fig. 1, conceptually, the direct heating mode has a positive component, serves to heat the whole troposphere, and is associated with a vertical shear flow. The second baroclinic mode is heated by the congestus heating, $H_{c}$, from below and by the stratiform
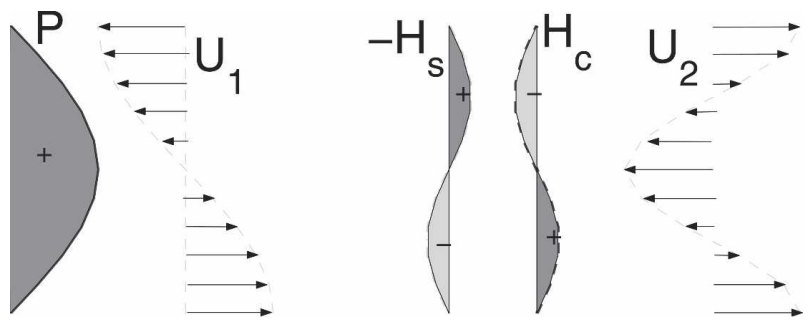

FIG. 1. Sketch of the two-baroclinic-mode model convective parameterization with three cloud types.

heating, $H_{s}$, from above and therefore cooled by $H_{c}$ from above and by $H_{s}$ from below. It is associated with a jet shear flow in the middle troposphere. The terms $S_{1}$ and $S_{2}$ are the radiative cooling rates associated with the first and second baroclinic modes, respectively.

The equations in (2.1) are obtained by a Galerkin projection of the hydrostatic primitive equations with constant buoyancy frequency onto the first two baroclinic modes. More details of their derivation are found in (Neelin and Zeng 2000; MS01; Frierson et al. 2004). In (2.1), $\mathbf{v}_{j}=\left(u_{j}, v_{j}\right)_{j=1,2}$ represent the first and second baroclinic velocities assuming $G(z)=\sqrt{2} \cos \left(\pi z / H_{T}\right)$ and $G(2 z)=\sqrt{2} \cos \left(2 \pi z / H_{T}\right)$ vertical profiles, respectively, while $\theta_{j}, j=1,2$ are the corresponding potential temperature components with the vertical profiles $G^{\prime}(z)=\sqrt{2} \sin \left(\pi z / H_{T}\right)$ and $2 G^{\prime}(2 z)=2 \sqrt{2} \sin (2 \pi z /$ $H_{T}$ ), respectively. Therefore, the total velocity field is approximated by

$$
\begin{aligned}
& \mathbf{V} \approx \overline{\mathbf{U}}+G(z) \mathbf{v}_{1}+G(2 z) \mathbf{v}_{2} \\
& w \approx-\frac{H_{T}}{\pi}\left[G^{\prime}(z) \operatorname{div} \mathbf{v}_{1}+\frac{1}{2} G^{\prime}(2 z) \operatorname{div} \mathbf{v}_{2}\right]
\end{aligned}
$$

where $\mathbf{V}$ is the horizontal velocity and $w$ the vertical velocity. The total potential temperature is given approximately by

$$
\Theta \approx z+G^{\prime}(z) \theta_{1}+2 G^{\prime}(2 z) \theta_{2} .
$$

Here $H_{T} \approx 16 \mathrm{~km}$ is the height of the tropical troposphere with $0 \leq z \leq H_{T}$ and $\mathbf{v}_{j}^{\perp}=\left(-v_{\boldsymbol{j}}, u_{\boldsymbol{j}}\right)$ while $\overline{\mathbf{U}}$ is the incompressible barotropic wind, which is set to zero here for the sake of simplicity.

For simplicity, the nonlinear interactions between the first and second baroclinic modes are ignored but they can be easily derived and incorporated into the equations (Majda and Biello 2003; Khouider and Majda 2005a,b). Also the Coriolis terms on the left-hand side of the momentum equations, $\beta y \mathbf{v}_{j}^{\perp}$, are dropped below thus eliminating the meridional features of the tropical disturbances leaving only moist gravity waves. Nevertheless, this turns out to be enough for capturing the 
convectively coupled Kelvin waves that have no meridional velocity and propagate along the equator.

The system of equations in (2.1) is augmented by an equation for the boundary layer equivalent potential temperature, $\theta_{\mathrm{eb}}$, and another for the vertically integrated moisture content, $q$;

$$
\begin{gathered}
\frac{\partial \theta_{\mathrm{eb}}}{\partial t}=\frac{1}{h_{b}}(E-D) \\
\frac{\partial q}{\partial t}+\overline{\mathbf{U}} \cdot \nabla q+\operatorname{div}\left[\left(\mathbf{v}_{1}+\tilde{\delta} \mathbf{v}_{2}\right) q\right]+\tilde{Q} \operatorname{div}\left(\mathbf{v}_{1}+\tilde{\lambda} \mathbf{v}_{2}\right) \\
=-P+\frac{1}{H_{T}} D .
\end{gathered}
$$

In (2.2), $h_{b} \approx 500 \mathrm{~m}$ is the height of the moist boundary layer while $\tilde{Q}, \tilde{\lambda}$, and $\tilde{\delta}$ are parameters associated with a prescribed moisture background and perturbation vertical profiles. According to the first equation in (2.2), $\theta_{\mathrm{eb}}$ changes in response to the downdrafts, $D$, and the sea surface evaporation $E$. The troposphere moisture equation for $q$ is derived from the bulk water vapor budget equation by imposing a moisture stratificationlike background vertical profile $q^{\text {tot }}=Q(z)+q$. The details of this derivation are reported in appendix A. The approximate numerical values of $\tilde{\lambda}=0.8$ and $\tilde{\delta}=0.1$ follow directly from the derivation, while the coefficient $\tilde{Q}$ arises from the background moisture gradient. We use the standard value $\tilde{Q} \approx 0.9$ (Yano and Emanuel 1991; Frierson et al. 2004).

In full generality, the parameterizations in (2.1) and (2.2) automatically have conservation of an approximation to vertically integrated moist static energy. Notice that, the precipitation rate in (2.2), balances the vertical average of the total convective heating rate in (2.1), therefore leading to the conservation of the vertical average of the equivalent potential temperature $\left\langle\theta_{e}\right\rangle=$ $\langle Q(z)\rangle+q+\langle\Theta\rangle+\left(h_{b} / H_{T}\right) \theta_{\mathrm{eb}}$ when the external forces, namely, the radiative cooling rates, $S_{1}, S_{2}$, and the evaporative heating, $E$, are set to zero. Also note that the sensible heating flux has been ignored in (2.1) for simplicity since this is a relatively small contribution in the Tropics. Here and elsewhere in the text $\langle f\rangle \equiv$ $\left(1 / H_{T}\right) \int_{0}^{H_{T}} f(z) d z$.

The equations in (2.1) and (2.2) for the prognostic variables $q, \theta_{\mathrm{eb}}, \theta_{j}, \mathbf{v}_{j}, j=1,2$, are written in nondimensional units where the equatorial Rossby deformation radius, $L_{e} \approx 1500 \mathrm{~km}$ is the length scale, the first baroclinic dry gravity wave speed, $c \approx 50 \mathrm{~m} \mathrm{~s}^{-1}$, is the velocity scale, $T=L_{e} / c \approx 8 \mathrm{~h}$ is the associated time scale, and the dry-static stratification $\bar{\alpha}=\left(H_{T} N^{2} \theta_{0} / \pi g\right) \approx$ $15 \mathrm{~K}$ is the temperature unit scale. The basic bulk parameters of the model are listed in Table 1 for the reader's convenience.
TABLE 1. Bulk constants in two-layer-mode model.

$\begin{aligned} & H_{T}= 16 \mathrm{~km}: \text { Height or the tropical troposphere } \\ & \tilde{Q}= 0.9: \text { Moisture stratification factor } \\ & \tilde{\lambda}= 0.8: \text { Second baroclinic relative contribution to the } \\ & \text { moisture convergence associated with the moisture } \\ & \text { background } \\ & \tilde{\delta}= 0.1: \text { Second baroclinic relative contribution to the } \\ & \text { moisture (nonlinear) convergence associated with the } \\ & \text { moisture anomalies } \\ & \tau_{R}= 75 \text { days: Rayleigh wind friction relaxation time } \\ & \tau_{D}= 50 \text { days: Newtonian cooling relaxation time } \\ & c_{d}= 0.001: \text { Boundary layer turbulence momentum friction } \\ & L_{e} \approx 1500 \mathrm{~km}: \text { Equatorial deformation radius, length scale } \\ & c \approx 50 \mathrm{~m} \mathrm{~s} \mathrm{~s}^{-1}: \text { Speed of the first baroclinic gravity wave } \\ & \text { velocity scale } \\ & T= L_{e} / c \approx 8 \text { h: Time scale } \\ & \bar{\alpha} \approx 15 \mathrm{~K}: \text { Dry static stratification, temperature scale } \\ & N= 0.01 \mathrm{~s}-1: \text { Brunt }- \text { Väisälä buoyancy frequency } \\ & \theta_{0}= 300 \mathrm{~K}: \text { Reference temperature } \\ & h_{b}= 500 \mathrm{~m}: \text { Boundary layer height } \\ & \bar{X}: \mathrm{RCE} \text { value of the variable } X \\ & \alpha_{2}= 0.1: \text { Relative contribution of } \theta_{2} \text { to the middle } \\ & \text { troposphere } \theta_{e}\end{aligned}$

\section{b. The convective parameterization}

The surface evaporative heating, $E$, in (2.2) obeys an adjustment equation toward the boundary layer saturation equivalent potential temperature, $\theta_{\mathrm{eb}}^{*}$,

$$
\frac{1}{h_{b}} E=\frac{1}{\tau_{e}}\left(\theta_{\mathrm{eb}}^{*}-\theta_{\mathrm{eb}}\right)
$$

with $\tau_{e}$ is the evaporative time scale. The value of $\theta_{\mathrm{eb}}^{*}$ on a warm ocean surface is fixed such that at radiative convective equilibrium we have $\theta_{\mathrm{eb}}^{*}-\bar{\theta}_{\mathrm{eb}}=10 \mathrm{~K}$, according to the Jordan sounding (Gill 1982).

Besides the second baroclinic moisture advection in (2.2), the originality of the present model resides in a new treatment of the deep convective heating/precipitation, $P$, and the downdrafts, $D$, as well as the introduction of the congestus heating, $H_{c}$, into the $\theta_{2}$ equation. The middle tropospheric equivalent potential temperature anomaly is defined approximately by

$$
\theta_{\mathrm{em}} \approx q+\frac{2 \sqrt{2}}{\pi}\left(\theta_{1}+\alpha_{2} \theta_{2}\right)
$$

where $\alpha_{2}=0.1$. Notice that the coefficient $2 \sqrt{2} / \pi$ in (2.4) results from the vertical average of the first baroclinic potential temperature, $\sqrt{2} \theta_{1} \sin \left(\pi z / H_{T}\right)$, while the small value for $\alpha_{2}$ adds a nonzero contribution from $\theta_{2}$ to $\theta_{\mathrm{em}}$ to include its contribution from the lower middle troposphere although its vertical average is zero.

Inspired by Zehnder (2001), we introduce and use a switch parameter $\Lambda$, which serves as a measure for the 
moistness and dryness of the middle troposphere. When the discrepancy between the boundary layer and the middle troposphere equivalent potential temperatures is above some fixed threshold, $\theta^{+}$, the atmosphere is defined as dry and we set $\Lambda=1$ and when this discrepancy is below some lower value, $\theta^{-}$, we have a relatively moist atmosphere and we set $\Lambda=\Lambda^{*}<1$. The lower threshold $\Lambda^{*}$ can basically take any value between zero and one, and here $\Lambda^{*}=0.2$ while $\Lambda$ is then interpolated (linearly) between these two values. More precisely, we set

$$
\Lambda=\left\{\begin{array}{ccc}
1 & \text { if } & \theta_{\mathrm{eb}}-\theta_{\mathrm{em}}>\theta^{+} \\
A\left(\theta_{\mathrm{eb}}-\theta_{\mathrm{em}}\right)+B & \text { if } & \theta^{-} \leq \theta_{\mathrm{eb}}-\theta_{\mathrm{em}} \leq \theta^{+} \\
\Lambda^{*} & \text { if } & \theta_{\mathrm{eb}}-\theta_{\mathrm{em}}<\theta^{-} .
\end{array}\right.
$$

Here $\theta^{+}=20 \mathrm{~K}$ and $\theta^{-}=10 \mathrm{~K}$ while $A$ and $B$ are fitting constants guaranteeing continuity of $\Lambda$. The value of $\theta^{-}$is chosen according to the Jordan sounding (Fig. 3.5 from Gill 1982). It represents a threshold below which the free troposphere is locally moist and accepts only deep convection.

Therefore, the precipitation, $P$, and the downdrafts, $D$, obey

$$
P=\frac{1-\Lambda}{1-\Lambda^{*}} P_{0} \quad \text { and } \quad D=\Lambda D_{0}
$$

while the stratiform and congestus heating rates, $H_{s}$ and $H_{c}$, solve the relaxation-type equations

$$
\frac{\partial H_{s}}{\partial t}=\frac{1}{\tau_{s}}\left(\alpha_{s} P-H_{s}\right)
$$

and

$$
\frac{\partial H_{c}}{\partial t}=\frac{t}{\tau_{c}}\left(\alpha_{c} \frac{\Lambda-\Lambda^{*}}{1-\Lambda^{*}} \frac{D}{H_{T}}-H_{c}\right),
$$

respectively. Notice that when the middle troposphere is dry, $\Lambda=1$, deep convection is completely inhibited, even if $P_{0}$, is positive, whereas congestus heating is favored. In the absence of deep convection, the downdrafts are interpreted as the subsidence associated with the detrainment of shallow clouds. In this sense, the shallow clouds serve to moisten and precondition the middle troposphere to sustain deep convection by lowering $\Lambda$ in the model via both the increase of $q$ and the decrease of $\theta_{\mathrm{eb}}$. The situation is inverted during the deep convective episodes when $\Lambda=\Lambda^{*}$. Moreover, the dry atmosphere increases the downdrafts, $D$, and promotes boundary layer clouds. This also is well reflected in the model.
TABLE 2. Parameters in the convective parameterization.

$\theta_{\mathrm{eb}}^{*}$ : Boundary layer saturation equivalent potential temperature

$\tau_{e} \approx 8 \mathrm{~h}$ or 9 days: Evaporative time scale in the boundary layer

$\theta^{ \pm}=10,20 \mathrm{~K}$ : Temperature thresholds used to define the switch function $\Lambda$

$\Lambda^{*}=0.2$ : Lower threshold of the switch function $\Lambda$

$A, B$ : Linear fitting constants interpolating the switch function $\Lambda$

$\tau_{s}=3 \mathrm{~h}$ : Stratiform heating adjustment time

$\alpha_{s}=0.25$ : Stratiform heating adjustment coefficient

$\tau_{c}=1 \mathrm{~h}$ : Congestus heating adjustment time

$\alpha_{c}=0.5$ (varies): Congestus heating adjustment coefficient

$a_{0}=7.5$ (varies): Inverse buoyancy time scale of convective parameterization

$a_{1}=0.1$ (varies): Relative contribution of $\theta_{e b}$ to the convective parameterization

$a_{2}=0.9$ (varies): Relative contribution of $q$ to the convective parameterization

$\tau_{\text {conv }}=2 \mathrm{~h}$ : Deep convective reference time scale

$\hat{q}$ : Threshold beyond which condensation takes place in

Betts-Miller scheme

$\gamma_{2}=0.1$ (varies): Relative contribution of $\theta_{2}$ to the convective parameterization (strength of lower troposphere coupling)

$\bar{\theta}_{\mathrm{eb}}-\bar{\theta}_{\mathrm{em}}=14 \mathrm{~K}$ : Discrepancy between boundary and middle tropospheric equivalent potential temperature at RCE.

$m_{0}$ (value is set by RCE): Scaling of downdraft mass flux

$\mu_{2}=0.5$ : Relative contribution of stratiform and congestus mass flux anomalies

The quantities $P_{0}$ and $D_{0}$ represent the maximum allowable deep convective heating/precipitation and downdrafts, respectively, independent of the value of the switch function $\Lambda$. Notice that conceptually the model is not bound to any type of convective parameterization. A Betts-Miller relaxation-type parameterization as well as a CAPE parameterization can be used to set up a closure for $P_{0}$. Indeed, here we use a combination of the two concepts by letting

$P_{0}=\frac{1}{\tau_{\text {conv }}}\left[a_{1} \theta_{\mathrm{eb}}+a_{2}(q-\hat{q})-a_{0}\left(\theta_{1}+\gamma_{2} \theta_{2}\right)\right]^{+}$,

where $\hat{q}$ is a threshold constant value measuring a significant fraction of the tropospheric saturation and $\tau_{\text {conv }}$ is the convective time scale while $a_{1}, a_{2}, a_{0}$ are nondimensional parameters specified below and in Table 2 (Fuchs and Raymond 2002; Frierson et al. 2004). In particular, the coefficient $a_{0}$, which is related to the inverse buoyancy relaxation time of Fuchs and Raymond (2002), is an important parameter to vary. The parameter $\gamma_{2}$, which couples $\theta_{2}$ to $P_{0}$, is also varied to assess the effects of the lower troposphere temperature variation on the parameterizations; a relatively warm lower troposphere will promote evaporation and de- 
trainment of cumulus clouds. Thus, it should result in a weakening of the deep convection.

The downdrafts are closed by

$$
D_{0}=\frac{m_{0}}{\bar{P}}\left[\bar{P}+\mu_{2}\left(H_{s}-H_{c}\right)\right]^{+}\left(\theta_{\mathrm{eb}}-\theta_{\mathrm{em}}\right),
$$

where $m_{0}$ is a scaling of the downdraft mass flux and $\bar{P}$ is a prescribed precipitation/deep convective heating at radiative convective equilibrium. Here $\mu_{2}$ is a parameter allowing for stratiform and congestus mass flux anomalies (MS01; M04). Finally the radiative cooling rates, $S_{1}, S_{2}$ in (2.1) are given by a simple Newtonian cooling model

$$
S_{j}=-Q_{R, j}^{0}-\frac{1}{\tau_{D}} \theta_{j}, \quad j=1,2,
$$

where $Q_{R, j}^{0}, j=1,2$ are the radiative cooling rates at $\mathrm{RCE}$. The basic constants in the model convective parameterization and the typical values utilized here are given in Table 2.

The equations in (2.1) through (2.11) describe the multicloud convective parameterization utilized here with characteristic features, combining those of convergence-driven and quasi-equilibrium schemes, briefly summarized below:

1) The parameterization respects conservation of vertically integrated moist static energy by design.

2) Low-level moistening from moisture convergence: the role of the second baroclinic convergence in the q equation in (2.2) is to increase locally the moisture content yielding a preconditioning of the atmosphere to sustain and trigger deep convection by moistening as well as decreasing the value of the switch function $\Lambda$ in (2.5).

3) Also from (2.5), no deep convection is allowed if the upper troposphere is dry even if $P_{0}$, is positive. This is reminiscent for the situation in nature where deep convection is inhibited even if CAPE is positive.

4) The deep convection is handled in a quasiequilibrium scheme framework with the three major contributors, $\theta_{\mathrm{eb}}, q$, and $\theta_{1}+\gamma_{2} \theta_{2}$. Deep convection being favored by the increase of $\theta_{\mathrm{eb}}$ and $q$, and the decrease of $\theta_{1}+\gamma_{2} \theta_{2}$, that is, a more moist and warmer boundary layer, as in CAPE parameterizations, a moist troposphere, as in Betts-Miller-type schemes, and a cooler lower middle troposphere, which favors saturation at weaker mixing ratios. The parameterization has the following dynamical effects on the variables: A) $\theta_{\mathrm{eb}}$ increases from the surface evaporative forcing, $E$, when the downdrafts, $D$, on the boundary layer are decreasing; B) $q$ increases from first and second baroclinic convergence and from evaporation of shallow clouds when the upper troposphere is dry, that is, $\Lambda=1$, reflected by the positive effect from the downdrafts, $D$, while $q$ decreases from deep convection which generates precipitation; and C) the combination $\theta_{1}+\gamma_{2} \theta_{2}$ increases after the deep convective warming episodes, which automatically induces weaker downdrafts on the boundary layer.

5) When the atmosphere is not extremely moist, $\Lambda>$ $\Lambda^{*}$, that is, during weak or vanishing deep convection episodes, a fraction $\alpha_{c}$ of the shallow convective activity is converted into congestus-precipitating clouds, via the factor $\left(\Lambda-\Lambda^{*}\right) D /\left(1-\Lambda^{*}\right) H_{T}$ in (2.8), which serves to increase $\theta_{2}$, inducing a decrease of $P_{0}$ as well as a decrease of the effective downdrafts $D$ by increasing the middle troposphere equivalent potential temperature.

6) For $\mu_{2}>0$, the negative anomalies in the stratiform mass flux (MS01; M04) help generate $\theta_{\mathrm{eb}}$ and restore CAPE by decreasing the effective downdrafts $D$.

7) When the parameters $\alpha_{2}, \gamma_{2}$, and $\mu_{2}$ in (2.4), (2.9), and (2.10), respectively, together with $\tilde{\delta}, \tilde{\lambda}$ in (2.2) are set to zero, the second baroclinic mode decouples completely from the convective parameterization and becomes slaved to the first baroclinic mode.

\section{Radiative convective equilibrium and linearized equations}

A standard tool in understanding the basic properties of a convective parameterization is the linearized stability analysis at radiative convective equilibrium (Emanuel 1987; Yano et al. 1998; Majda and Shefter 2001a; MS01; Fuchs and Raymond 2002). A radiative convective equilibrium (RCE), which is a state where the convective heating is balanced by the radiative cooling, is a time-independent, static, and spatially homogeneous solution to the set of Eqs. (2.1)-(2.11) described above. It sets up a steady-state solution around which convective waves can oscillate and grow. In this section, we construct such an RCE solution and perform a linear stability study for small wavelike perturbations from this RCE. Therefore, we let
(1) $\bar{E}=\bar{D}$
(2) $\frac{1}{H_{T}} \bar{D}=\bar{P}=\frac{1-\bar{\Lambda}}{1-\Lambda_{*}} \bar{P}_{0}$
(3) $\frac{\pi}{2 \sqrt{2}} \bar{P}=Q_{R, 1}^{0}$
(4) $\frac{\pi}{2 \sqrt{2}}\left(-\bar{H}_{s}+\bar{H}_{c}\right)=Q_{R, 2}^{0}$.
(5) $\alpha_{s} \bar{P}=\bar{H}_{s}$
(6) $\alpha_{c} \frac{\bar{\Lambda}-\Lambda_{*}}{1-\Lambda_{*}} \frac{\bar{D}}{H_{T}}=\bar{H}_{c}$ 
Notice that Eq. (2) in (3.1) implies that the RCE precipitation, $\bar{P}$, and the RCE downdraft, $\bar{D}$, are both zero if the upper troposphere is dry at equilibrium, that is, $\bar{\Lambda}=1$ and Eqs. (1) and (3) imply that both the evaporative forcing, $\bar{E}$, and the radiative cooling, $Q_{R, 1}^{0}$, should also be zero in this case. Such purely congestus equilibria are believed nonphysical and are therefore discarded.

An RCE solution is completely determined by fixing the evaporative rate $\bar{E}$ alone provided the state of the upper troposphere is also specified by fixing $\bar{\Lambda}$ or equivalently $\bar{\theta}_{\mathrm{eb}}-\bar{\theta}_{\mathrm{em}}$. With the value $\theta_{\mathrm{eb}}^{*}-\bar{\theta}_{\mathrm{eb}}=10 \mathrm{~K}$, according to the Jordan tropical sounding (Gill 1982), the realistic value of radiative cooling, $Q_{R, 1}^{0}$, given by 1 $\mathrm{K}$ day $^{-1}$ yields a boundary layer evaporative time scale $\tau_{e} \approx 8 \mathrm{~h}$ and this is the standard value utilized below in section 4. The weaker value of $Q_{R, 1}^{0}=0.04 \mathrm{~K} \mathrm{day}^{-1}$ and $\tau_{e} \approx 9$ days is also used below for comparison purposes.

The linearized equations about an RCE solution are then obtained for the first-order perturbation, $U(x, t)=$ $\left(u_{1}, u_{2}, \theta_{1}, \theta_{2}, \theta_{\mathrm{eb}}, q, H_{s}, H_{c}\right)$, and the explicit formulation of the linear system is presented in appendix $\mathrm{B}$. Next, we look for traveling wave solutions for the linearized system with the form $U(x, t)=U \exp [i(k x-$ $\omega t)$ ]. Here $k$ is the wavenumber and $\omega=\omega(k)$ is the generalized dispersion relation where $\operatorname{Re}(\omega) / k$ is the phase speed and $\operatorname{Im}(\omega)$ is the growth of the linear wave (Majda and Shefter 2001a; MS01; M04). Because it is impossible to get explicitly the analytic expressions for the dispersion relations associated with the $8 \times 8$ system, the corresponding eigenvalue problem is solved numerically on a computer. The detailed results are given in section 4 below.

\section{Linear stability: Results and analysis}

A lot of parameters are involved in the present parameterization and the model stability is sensitive to many of them. Nevertheless, plausible values were derived by physical and/or mathematical consistency arguments for the majority of the parameters during either the derivation of the model in section 2 or the setup of the RCE solution in section 3. Only a few parameters need to be systematically varied. These include $a_{0}, a_{1}, a_{2}$, and $\gamma_{2}$ in (2.9), and $\bar{\theta}_{\mathrm{eb}}-\bar{\theta}_{\mathrm{em}}$, which also fixes $\bar{\Lambda}$, as well as the congestus coefficient $\alpha_{\mathrm{c}}$ in (2.8). Moreover, the constants $\tilde{\lambda}=0.8, \alpha_{2}=0.1$, and $\mu_{2}=0.5$ are also set to zero below to assess the effect of coupling of the second baroclinic mode.

\section{a. Homogeneous stability of the RCE}

A stability analysis for the homogeneous state RCE, which is represented by the linear mode solutions cor-
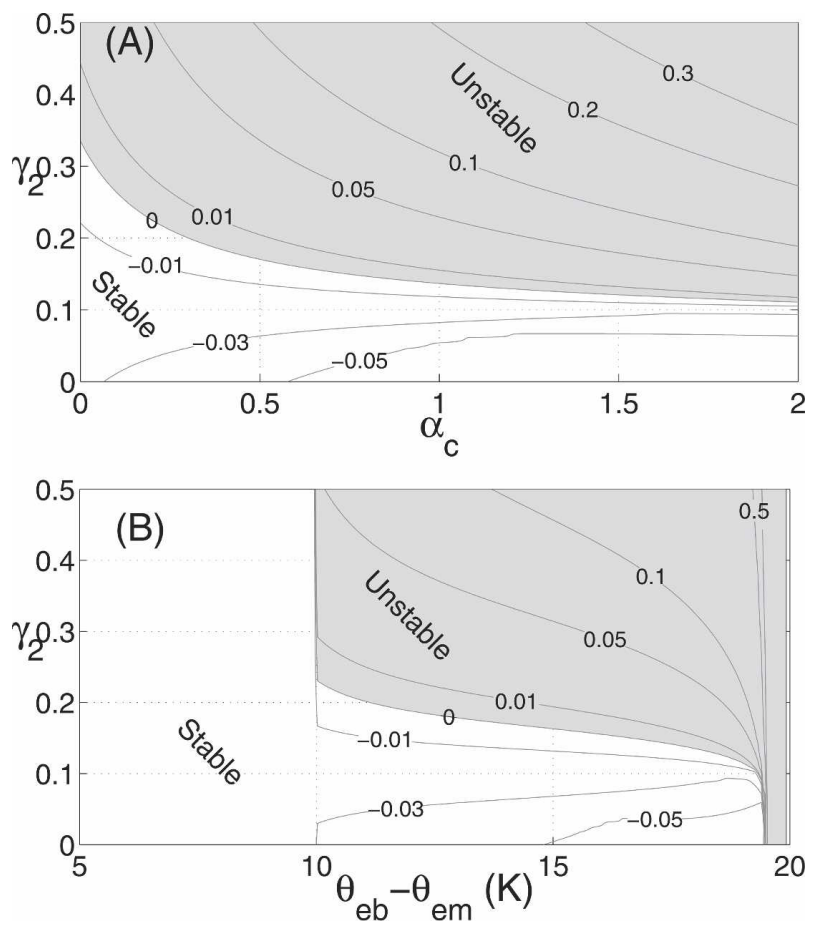

FIG. 2. Bifurcation diagram for the homogeneous state RCE (a) in the $\gamma_{2}-\alpha_{c}$ plane for fixed $\bar{\theta}_{\mathrm{eb}}-\bar{\theta}_{\mathrm{em}}=14 \mathrm{~K}$ and (b) in the $\gamma_{2}-$ $\bar{\theta}_{\mathrm{eb}}-\bar{\theta}_{\mathrm{em}}$ plane for fixed $\alpha_{c}=0.5$. The regions of positive maximum growth among the $k=0$ modes are shaded and a few contours are plotted.

responding to wavenumber $k=0$ is presented here. The corresponding bifurcation diagrams for fixed $\bar{\theta}_{\mathrm{eb}}-$ $\bar{\theta}_{\mathrm{em}}=14 \mathrm{~K}$ and for fixed $\alpha_{\mathrm{c}}=0.5$ are reported in Figs. $2 \mathrm{a}, \mathrm{b}$, in the $\gamma_{2}-\alpha_{\mathrm{c}}$ and $\gamma_{2}-\bar{\theta}_{\mathrm{eb}}-\bar{\theta}_{\mathrm{em}}$ planes, respectively, for the realistic radiative cooling of $Q_{R, 1}^{0}=$ $1 \mathrm{~K} \mathrm{day}^{-1}$. The two pictures in Figs. 2a,b are representative of the stability behavior when the three parameters, $\gamma_{2}, \alpha_{c}, \bar{\theta}_{\mathrm{eb}}-\bar{\theta}_{\mathrm{em}}$ are varied. For a mixed RCE, $10 \mathrm{~K}=\theta^{-}<\bar{\theta}_{\mathrm{eb}}-\bar{\theta}_{\mathrm{em}}<\theta^{+}=20 \mathrm{~K}$, a $k=0$ mode turns unstable when the parameter $\gamma_{2}$ exceeds some value around 0.4 or lower, independent of $\alpha_{c}$ whereas the homogeneous state RCE is stable and weakly damped when $\gamma_{2} \leq 0.1$, for all $0 \leq \alpha_{c} \leq 2$ except for a small band near $20 \mathrm{~K}$. This band is very narrow when $\alpha_{c} \leq 0.5$ but it expands toward the interior as $\alpha_{c}$ grows. This homogeneous state RCE instability is not too sensitive to changes in the values of the convective parameters $a_{0}$, $a_{1}, a_{2}$.

As demonstrated in Fig. 2b, the homogeneous state is stable, for all values of other parameters when the RCE is set to the purely deep convective state, $\bar{\theta}_{\mathrm{eb}}-\bar{\theta}_{\mathrm{em}}<$ $\theta^{-}$. Also, although not shown here, when $Q_{R, 1}^{0}=0.04$ $\mathrm{K} \mathrm{day}^{-1}$, the region of stability expands toward larger values of $\gamma_{2}$ such that with $\alpha_{c}=0.5$ the instability is confined to a narrow strip near $\bar{\theta}_{\mathrm{eb}}-\bar{\theta}_{\mathrm{em}}=20 \mathrm{~K}$ for all 
$0 \leq \gamma_{2} \leq 1$. Taken together, all these results indicate that the RCE is unstable to homogeneous perturbations for $\bar{\Lambda}$ close to one in reasonable parameter regimes, that is, for RCE's dominated by congestus behavior and stable for deep convective dominated RCEs.

The linear stability analysis involving nonzero wavenumbers, $k \geq 1$, is addressed next. We use $\alpha_{c}=0.5$, $\gamma_{2}=0.1, \bar{\theta}_{\mathrm{eb}}-\bar{\theta}_{\mathrm{em}}=14 \mathrm{~K}$, with the radiative cooling $Q_{R, 1}^{0}=1 \mathrm{~K} \mathrm{day}^{-1}$ in our standard set of parameters. According to the analysis conducted above this constitutes a parameter regime whose homogeneous state $\mathrm{RCE}$ is stable.

\section{b. Basic stability diagram for various parameter regimes}

In Fig. 3, the growth rate and phase speed as functions of the wavenumber, $k \geq 0$, are presented for the standard RCE with $\bar{\theta}_{\mathrm{eb}}-\bar{\theta}_{\mathrm{em}}=14 \mathrm{~K}$ for the standard parameter values, $Q_{R, 1}^{0}=1 \mathrm{~K}$ day $^{-1}, \alpha_{c}=0.5, \gamma_{2}=0.1$, $a_{0}=7.5, a_{1}=0.1, a_{2}=0.9$ (the standard case) and are also presented for the other values, $\alpha_{c}=2$ with the remaining parameters fixed as well as the weaker radiative cooling $Q_{R, 1}^{0}=0.04 \mathrm{~K} \mathrm{day}^{-1}$ with $\gamma_{2}=1$ and for purely deep convective RCE, $\bar{\theta}_{\mathrm{eb}}-\bar{\theta}_{\mathrm{em}}=9 \mathrm{~K}$.

These four cases show that, in the parameter regimes where the $k=0$ mode is stable, the basic instability in the model parameterization consists of moist traveling waves with speeds in the regime 20 to $15 \mathrm{~m} \mathrm{~s}^{-1}$ basically confined to horizontal scales larger than equatorial synoptic scales around roughly $3000 \mathrm{~km}$. The bands of instability show remarkably little sensitivity to either the radiative cooling rate $Q_{R, 1}^{0}$ or the congestus coefficient $\alpha_{c}$ while the effect of increasing $\gamma_{2}$ from 0.1 to 1 creates a larger band of unstable wavelengths still confined to the large scales. However, the growth rate of the instability changes moderately over all these parameter variations in the four cases shown here. As shown in Table 3, the effect of changing the inverse buoyancy time-scale parameter $a_{0}$ on the stability diagram is that increasing $a_{0}$ confines the values of the instability to increasingly larger spatial scales and decreases the growth rates at the same time. The system is stable for values of $a_{0}$ slightly larger than 10.5 , when the other parameters are kept as in the standard case. All of these results indicate that even though the basic parameterization depends on low-level moisture convergence, there are no wave-CISK instabilities at small scales in the basic parameterization for the standard RCE.

For RCEs with higher values of $\bar{\theta}_{\mathrm{eb}}-\bar{\theta}_{\mathrm{em}}$, for which the homogeneous state is already unstable, the stability and phase diagrams corresponding to Fig. 3 (results not shown) bifurcate drastically and display a remarkably complex behavior with the emergence of other moisture waves, when compared to the standard case in Fig. 3a. It starts by the appearance, at the mesoscopic scales around $400 \mathrm{~km}$, of a small band of instability of moist gravity waves moving at the second baroclinic dry gravity wave speed of $25 \mathrm{~m} \mathrm{~s}^{-1}$ and a pair of standing modes unstable at the planetary scales, in addition to the 15 to $20 \mathrm{~m} \mathrm{~s}^{-1}$ basic moist gravity wave instability. When $\bar{\theta}_{\mathrm{eb}}-\bar{\theta}_{\mathrm{em}}$ is increased further the $25 \mathrm{~m} \mathrm{~s}^{-1}$ band of instability expands toward the synoptic scales while the basic moist gravity waves instability expands toward small (synoptic and mesoscopic) scales with the phase speeds asymptoting to zero at these small scales. However, at the limit $\bar{\theta}_{\mathrm{eb}}-\bar{\theta}_{\mathrm{em}} \approx 20 \mathrm{~K}$ only one standing mode survives with constant growth rates at small scales. The details of these bifurcations as well as the structures of theses waves will be reported in Khouider and Majda (2005c).

\section{c. Instability of limiting parameter regimes}

To elucidate the role of the second baroclinic coupling and especially the low-level moisture convergence, we report here the stability results when the parameters $\alpha_{2}, \gamma_{2}, \mu_{2}, \tilde{\lambda}$ are separately set to zero while the other parameters are kept as in the standard case. In Fig. $4 \mathrm{a}$, we have $\tilde{\lambda}=0$ so that the second baroclinic convergence is suppressed from the moisture equation in (2.2). Suddenly, the basic moist gravity wave instability disappears and it is replaced by a standing mode with a band of instability expanding to small scales and the growth rates are asymptotically constant at small scales. A similar stability diagram is obtained when both $\tilde{\lambda}$ and $\mu_{2}$ are set to zero but with much weaker growth rates and a band of instability significantly shifted toward the synoptic scales, as depicted in Fig. 4c. When $\tilde{\lambda}=0$, the standing mode instability diagram has very little sensitivity to the parameters $\alpha_{2}, \gamma_{2}$ for both cases $\mu \neq 0$ and $\mu=0$, respectively. Especially for the latter case, it is not surprising to see that the convectively coupled gravity wave instability disappears since WISHE is suppressed (MS01; M04). However, the emergence of a new standing mode with a range of instability expanding to small scales at an asymptotically constant growth is a different phenomenon. The instability diagram resembles the one encountered in one baroclinic mode models with moisture radiative feedback (Fuchs and Raymond 2002) and the associated mode is reminiscent of the nearly neutrally stable moisture mode present in quasi-equilibrium parameterizations with a single vertical mode (Neelin and $\mathrm{Yu}$ 1994; Majda and Shefter 2001a).

In Fig. $4 \mathrm{~b}$, we set $\gamma_{2}=0$ and switch back $\tilde{\lambda}$ and $\mu_{2}$ to 

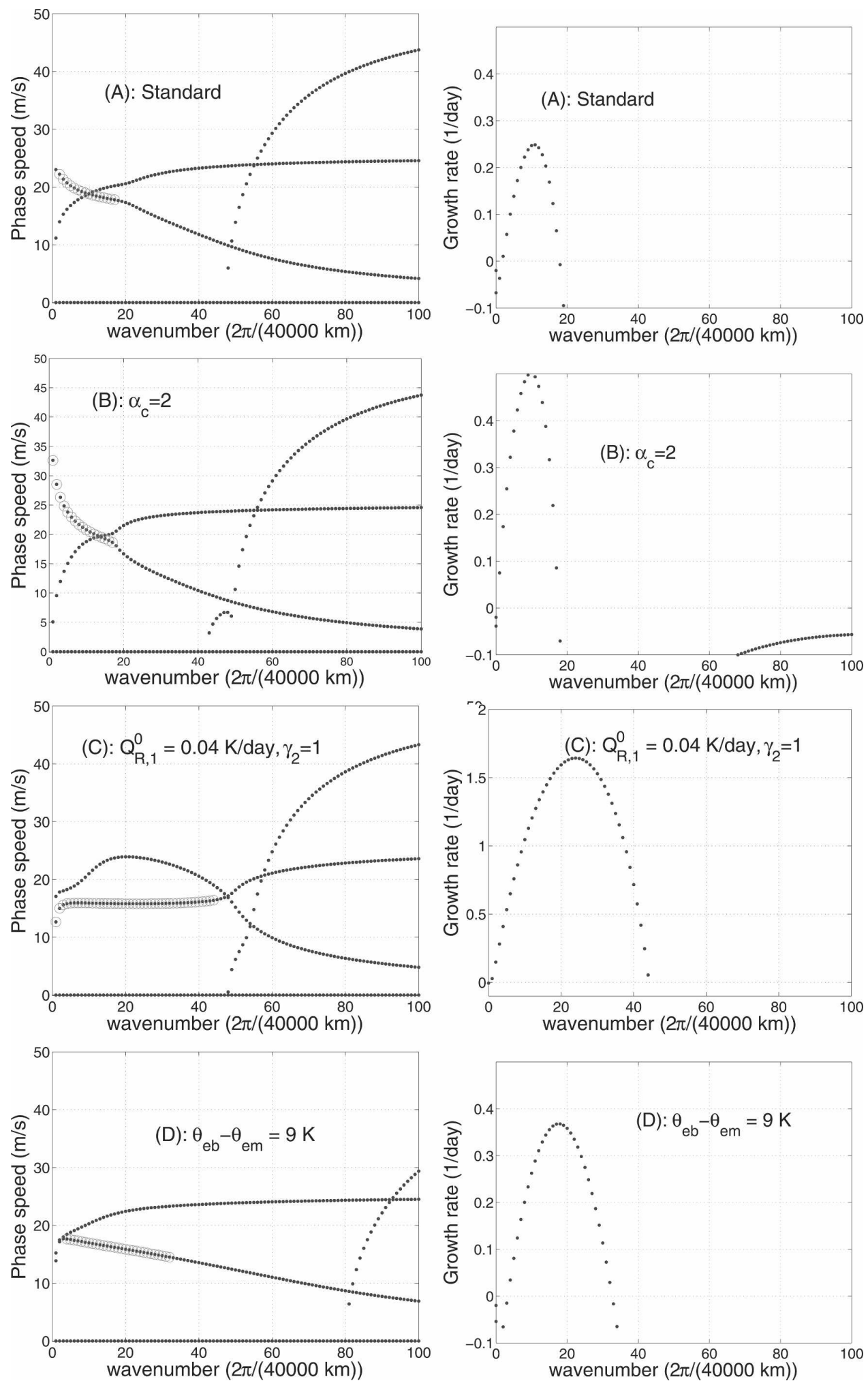

FIG. 3. (left) Phase speed and (right) growth rate as functions of the wavenumber for (a) the standard parameter values (see text), (b) when $\alpha_{c}=2$, (c) when $Q_{R, 1}^{0}=0.04 \mathrm{~K} \mathrm{day}^{-1}$ and $\gamma_{2}=1$ (note the change in growth scale), and (d) when $\bar{\theta}_{\mathrm{eb}}-\bar{\theta}_{\mathrm{em}}=9 \mathrm{~K}$. Because of symmetry only the first quadrant is shown for the dispersion relation diagram and the branches with positive growths are highlighted with small circles. 
TABLE 3. Range of instability and growth rates when the inverse buoyancy time parameter $a_{0}$ varies.

\begin{tabular}{lcc}
\hline \hline$a_{0}$ & $\begin{array}{c}\text { Range of unstable } \\
\text { wavenumbers }\end{array}$ & $\begin{array}{c}\text { Maximum growth } \\
\text { rate }\left(1 \text { day }^{-1}\right)\end{array}$ \\
\hline 5 & $2 \leq k \leq 26$ & 0.63 \\
7.5 & $2 \leq k \leq 17$ & 0.25 \\
10 & $3 \leq k \leq 7$ & 0.026 \\
11 & Stable & -0.006 \\
\hline
\end{tabular}

their standard values. The standing mode instability is eliminated (by $\tilde{\lambda}$ being not zero) while the basic moist gravity wave instability shrinks toward the planetary scales with significantly weaker growths when com- pared to the standard case in Fig. 3. In addition, when $\tilde{\lambda} \neq 0$, for both cases $\gamma_{2}=0.1$ and $\gamma_{2}=0$, the basic gravity wave instability has very little sensitivity to the parameters $\mu_{2}, \alpha_{2}$.

We conclude from the above that the second baroclinic low-level moisture convergence plays a major role in the generation of the basic moist gravity wave instability as well as filtering the standing mode instability while the lower middle tropospheric coupling is important for the amplification of this instability. Moreover, when $\tilde{\lambda}=0$, the stratiform and congestus mass flux contribution to the downdrafts amplifies the growth of the standing mode.

A change in parameters toward "a CAPE regime"
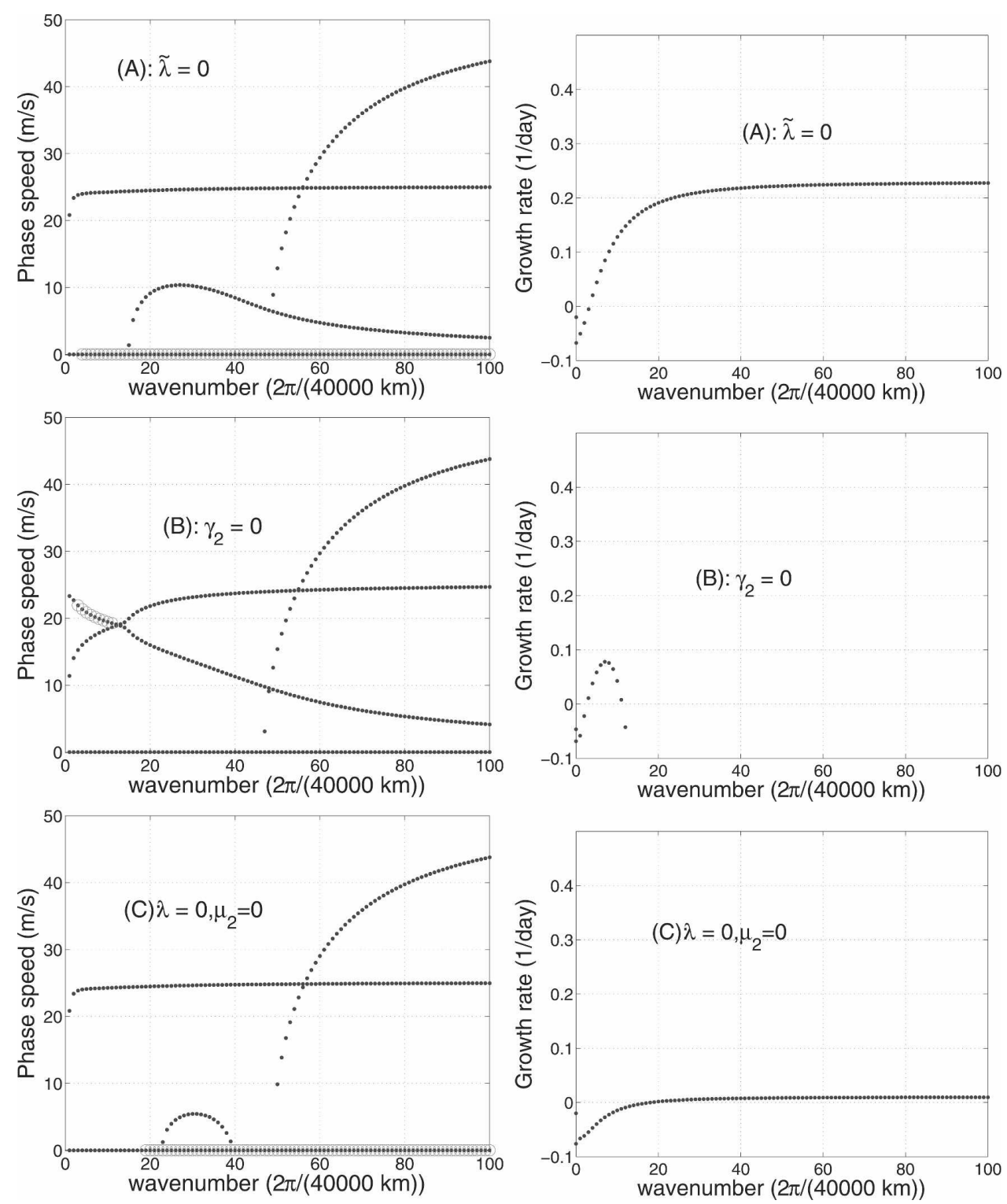

FIG. 4. Same as Fig. 3 but for the limiting parameter regimes (a) $\tilde{\lambda}=0$, (b) $\gamma_{2}=0$, and (c) $\tilde{\lambda}=0, \mu_{2}=0$. 


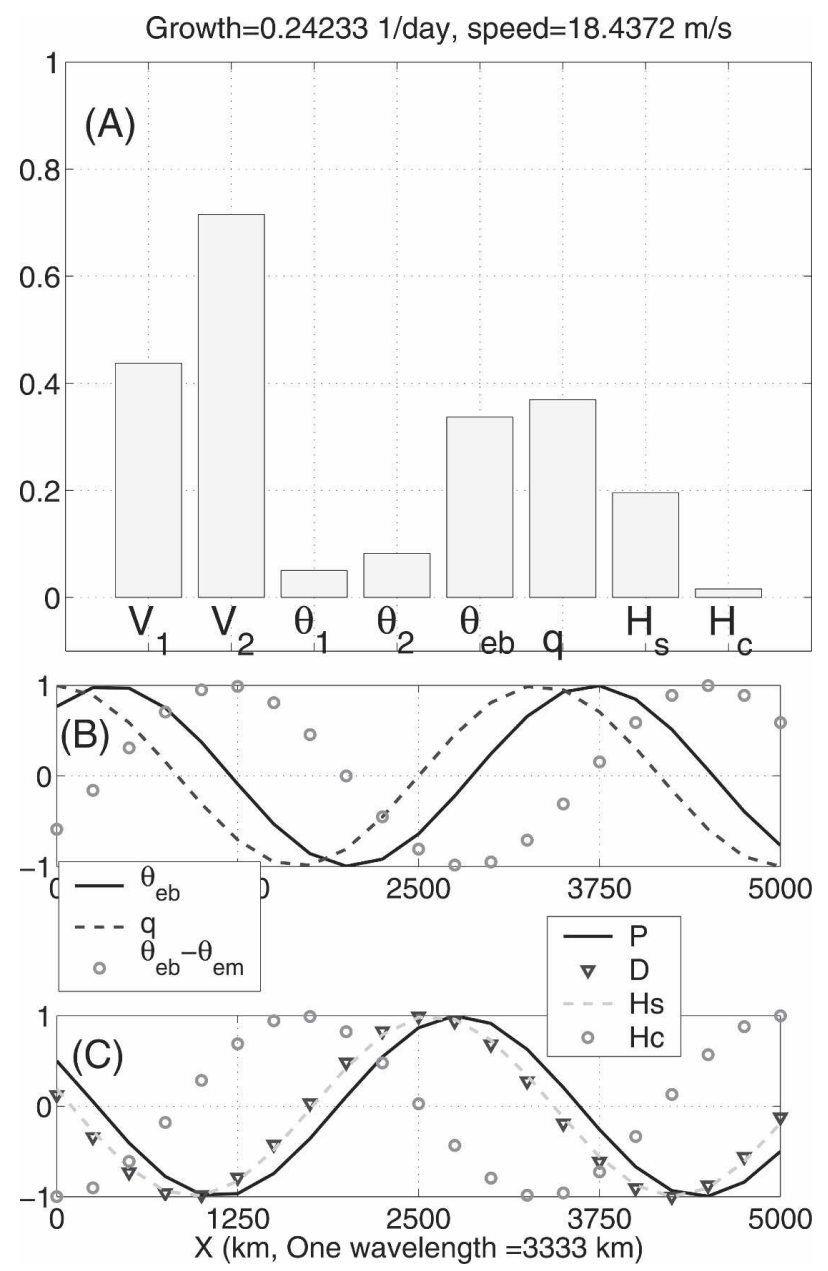

FIG. 5. Physical and dynamical structure of a convectively coupled wave for the standard case in Fig. 3 at wavenumber $k=$ 12. (a) Bar diagram showing the strength of prognostic components, (b) zonal structure of the moist thermodynamic variables, and (c) zonal structure of convective heating and downdrafts. The zonal structure plots are normalized to unity to fit on a same figure.

favoring boundary layer $\theta_{e}$ contributions in (2.9) is facilitated by setting $a_{1}=0.9, a_{2}=0.1$ with other parameters the same as the standard case. Although not reported in detail here this leads to a significant decrease in the growth rates, an increase of the phase speeds of the unstable waves at the synoptic scales, and a slight stretching of the band of instability toward small scales, when compared to the standard case in Fig. 3.

\section{Physical structure of the unstable waves}

In this section we look at the dynamic features and the detailed zonal and vertical structure of the unstable moist convectively coupled waves identified in the lin-
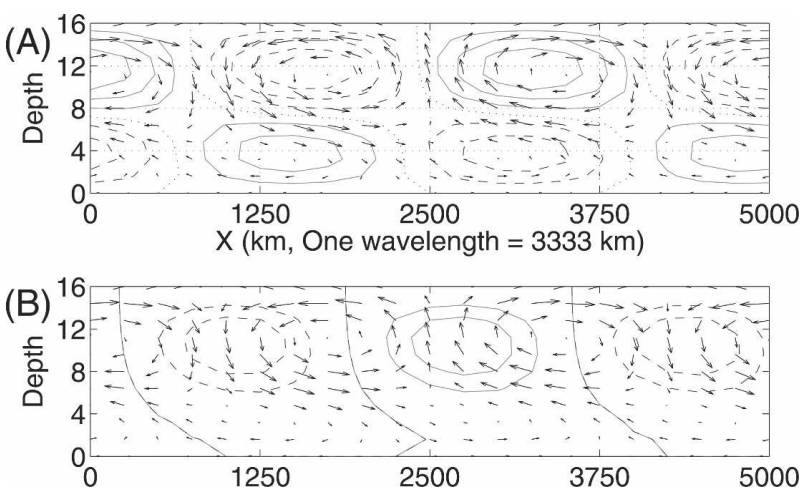

FIG. 6. Same as in Fig. $5 ; x-z$ contours of (a) the total potential temperature anomalies and (b) the total convective heating anomalies. Solid $=$ positive anomalies and dashed $=$ negative anomalies with the flow field profile (arrows) overlaid.

earized stability analysis in section 4 . Results are presented for the standard parameter values, $\gamma_{2}=0.1, \alpha_{c}=$ 0.5 with radiative cooling $Q_{R, 1}^{0}=1 \mathrm{~K}^{0}$ day $^{-1}$ with the standard RCE, $\bar{\theta}_{\mathrm{eb}}-\theta_{\mathrm{em}}=14 \mathrm{~K}$. Bar diagrams of the relative strength of the variables, $u_{1}, u_{2}, \theta_{1}, \theta_{2}, \theta_{\mathrm{eb}}, q, H_{s}$, $H_{c}$ for the corresponding unstable eigenvectors (MS01; M04) are utilized below.

In Fig. 5, the bar diagram for the unstable eigenvector and the structure of the anomalies in important physical quantities is presented through a one-and-ahalf spatial period cycle for the eastward-moving moist gravity wave with phase speed of $18 \mathrm{~m} \mathrm{~s}^{-1}$ and a wavelength of $3333 \mathrm{~km}(k=12)$ corresponding to the unstable mode for the standard case depicted in Fig. 3a. It is apparent from the bar diagram in Fig. 5a that all physical variables have significant magnitudes in the unstable wave. From Fig. 5c, the maximum in the anomaly for $P$ occurs at roughly $2700 \mathrm{~km}$ and signifies the location of maximum deep convection in the wave. This maximum is preceded by a maximum of congestus heating at $5000 \mathrm{~km}$ located in the dry anomaly phase of the wave and a maximum of moisture anomalies at 3300 $\mathrm{km}$, which serves to precondition the wave for the deep convective episode. There are also maxima in the CAPE in the leading dry anomaly phase of the wave with a maximum for $\theta_{\mathrm{eb}}$ occurring at $3700 \mathrm{~km}$ (Fig. 5b). The moisture minimum at roughly $1700 \mathrm{~km}$ as well as its periodic image at $5000 \mathrm{~km}$ occurs from drying of the troposphere after the deep convective episode while $\theta_{\mathrm{eb}}$ has a minimum at $2000 \mathrm{~km}$ corresponding to the strong stratiform downdrafts that lag the deep convection and help to consume CAPE. All of these facets of the unstable moist gravity wave are in qualitative agreement with current observations for superclusters (Wheeler et al. 2000; Straub and Kiladis 2002).

In Fig. 6, the $x-z$ structure of (a) the potential tem- 


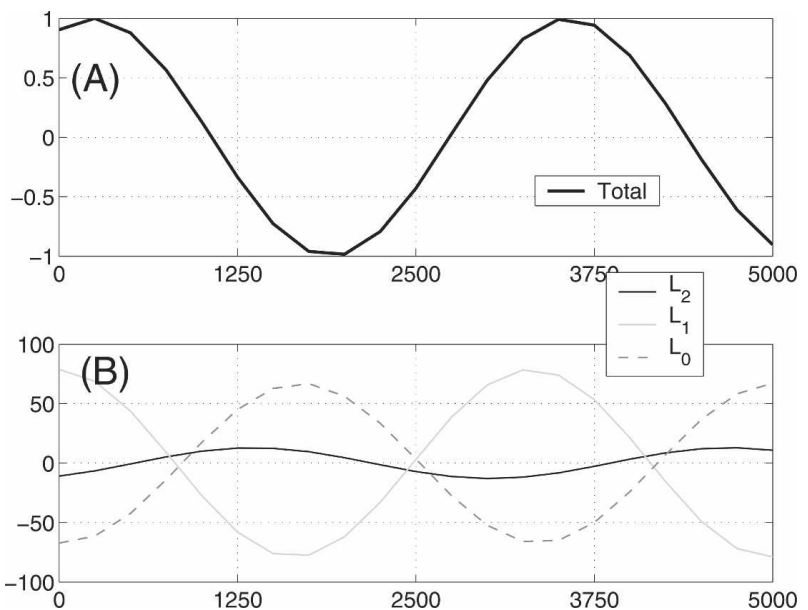

FIG. 7. Deep-convective heating budget. (a) Total tendency $(\partial P /$ $\partial t$ ) and (b) the contributions from the second baroclinic $\left(L_{2}\right)$, the first baroclinic $\left(L_{1}\right)$, and the moist thermodynamics $\left(L_{0}\right)$. [See Eq. (2.3).]

perature and (b) the total heating fields are depicted with the velocity structure overlaid. The physical structure of the wave also strongly resembles observations of convectively coupled Kelvin waves qualitatively with anomalous cold temperatures in the lower troposphere and warm temperatures in the upper troposphere within and slightly leading the heating region and strong updrafts in the wave and an upward and westward tilting structure with height (Wheeler et al. 2000; Straub and Kiladis 2002). Thus, as in MS01 and M04, besides the phase velocity, this unstable moist gravity wave reproduces key structural features of the moist convectively coupled waves from observations; one prominent new feature of the present models is the role of congestus heating and the subsequent tropospheric moistening in triggering the deep convective region in the moist gravity wave.

To confirm the dynamical intuition in Figs. 5 and 6 and discussed above for the moist eastward propagating unstable wave, we derive an equivalent of the CAPE budget equation used in MS01, by writing down the time derivative for the deep-convective heating $P$ :

$$
\begin{aligned}
\frac{\partial P}{\partial t}= & K_{1} \theta_{2}+K_{2} H_{s}+K_{3} \frac{\partial u_{2}}{\partial x}+K_{4} H_{c}+K_{5} q+K_{6} \theta_{\mathrm{eb}} \\
& +K_{7} \theta_{1}+K_{8} \frac{\partial u_{1}}{\partial x} .
\end{aligned}
$$

Here the $K_{i}$ s are constants that are easily obtained from the linearized system in (B1) appendix B. As in MS01, it is convenient to group together the terms in (5.1) depending on the second baroclinic mode, $\theta_{2}, u_{2}, H_{s}$, $H_{c}$, the moist thermodynamic variables, $q, \theta_{\mathrm{eb}}$, and the first baroclinic variables, $\theta_{1}, u_{1}$. Therefore (5.1) takes the form

$\frac{\partial P}{\partial t}=L_{2}\left(\theta_{2}, H_{s}, u_{2}, H_{c}\right)+L_{1}\left(\theta_{1}, u_{1}\right)+L_{0}\left(q, \theta_{\mathrm{eb}}\right)$.

In Fig. 7 a we plot the normalized total tendency, $(\partial P /$ $\partial t$ ), for the moist gravity wave. Notice that, the tendency plot in Fig. 7 is not perfectly correlated with the deep convective heating $P$ in Fig. 5c itself, but it is actually shifted to the right resulting in the eastward propagation of the moist gravity wave.

The contributions from $L_{2}, L_{1}$, and $L_{0}$ are shown in Fig. $7 \mathrm{~b}$ and show that for the moist gravity wave, the second baroclinic mode contributes to the first stage of the tendency, thus initiating the positive heating anomaly while the first baroclinic mode acts during the amplification stage. The thermodynamic variables, $q$ and $\theta_{\mathrm{eb}}$, do not seem to play a direct positive role in the deep convective heating tendency. They tend to counterbalance the first baroclinic tendency, which, as we know from section 4 above, would eliminate the moist gravity wave instability if the second baroclinic mode were absent. Indeed, $q$ and $\theta_{\mathrm{eb}}$ serve to precondition and destabilize the lower troposphere during the dry phase of the wave and somehow excite the second baroclinic mode via the congestus heating, which in turn triggers the instability.

In Table 4 we report the normalized correlation factors $\int(\partial P / \partial t) K_{j} X_{j} d x / \int(\partial P / \partial t)^{2} d t$ for the different terms on the right of (5.1) for the unstable moist gravity wave at different parameter regimes where $X_{j}$ denotes any

TABLE 4. Relative contributions to the convective heating budget [see Eq. (5.1)] for the moist gravity wave at different parameter

\begin{tabular}{|c|c|c|c|c|c|c|c|c|}
\hline Wave regime & $K_{1} \theta_{2}$ & $K_{2} H_{s}$ & $K_{3}\left(\partial U_{2} / \partial x\right)$ & $K_{4} H_{c}$ & $K_{5} q$ & $K_{6} \theta_{e b}$ & $K_{7} \theta_{1}$ & $\overline{K_{8}\left(\partial U_{1} / \partial x\right)}$ \\
\hline Standard & -9.0647 & -0.0118 & 1.8995 & 0.0042 & -57.8926 & -5.1404 & 69.3866 & 1.8191 \\
\hline$\alpha_{c}=0.25$ & -8.6267 & -0.0159 & 1.8808 & 0.0028 & -57.8121 & -5.2261 & 68.9397 & 1.8574 \\
\hline$\alpha_{c}=0.75$ & -9.4697 & -0.0072 & 1.9307 & 0.0039 & -58.0673 & -5.0405 & 69.8212 & 1.8288 \\
\hline$\alpha_{c}=2$ & -10.4977 & 0.0168 & 2.2493 & -0.0300 & -59.7521 & -4.5085 & 71.1242 & 2.3980 \\
\hline$\gamma_{2}=0(k=8)$ & 0.0603 & 0.0978 & 2.4156 & -0.0891 & -151.0954 & -10.8987 & 159.6775 & 0.8321 \\
\hline$\mu_{2}=0$ & -9.2692 & -0.1034 & 1.7497 & 0.0546 & -59.0541 & -3.4578 & 69.5055 & 1.5748 \\
\hline
\end{tabular}
regimes. The standard values $\gamma_{2}=0.1, Q_{R, 1}^{0}=1 \mathrm{~K} \mathrm{day}^{-1}, \mu_{2}=0.5, \alpha_{c}=0.5, k=12$ are used except when specified. 
variable in (5.1). Clearly, for the moist gravity waves, it is the low-level convergence and the congestus heating that contributes most of the second baroclinic positive tendency during the triggering stage, through the terms $K_{3}\left(\partial u_{2} / \partial x\right)$ and $K_{4} H_{c}$ while both $\theta_{1}$ and $\left(\partial u_{1} / \partial x\right)$ contribute during the amplification stage. Both $q$ and $\theta_{\mathrm{eb}}$ overall contribute negatively to the total tendency. From Table 4, a significant deviation from the standard value $\alpha_{c}=0.5$ decreases the positive contribution of the congestus heating $H_{c}$ to the total tendency, which in some perspective makes this standard choice optimal. The extreme value of $\alpha_{c}=2$ makes the congestus heating contribute negatively to the convective heating tendency, this in essence reflects the fact that congestus clouds compete with deep convection by removing moisture from the lower troposphere. From Table 4, when $\gamma_{2}=0$ the second baroclinic mode contributes to the convective parameterization through the downdrafts and to the switch function, directly and via $\theta_{\mathrm{em}}$. This is overall a positive effect on the deep convective tendency. When $\mu_{2}=0$, the contribution of $K_{3} H_{s}$ in Table 4 decreases, which implies that overall the stratiform mass flux contribution to the downdrafts favors the deep convective tendency.

\section{Concluding discussion}

A multicloud intermediate model convective parameterization, within the framework of two vertical baroclinic modes (TVBM), such as in Mapes (2000) and Majda and Shefter (2001b), is developed in section 2. The first mode is heated by deep convective clouds, as in early TVBM models, while the second mode is heated by stratiform clouds (from above) and cumulus congestus clouds (from below). Besides the introduction of the third type of clouds into the TVBM models, an equation for the vertically averaged moisture content with both a first and a second baroclinic convergence is systematically derived and used, the second baroclinic part ensuring a low-level convergence of moisture. Moreover, the model relies on a nonlinear switch function $\Lambda$ measuring the moistness and dryness of the troposphere (Zehnder 2001) to favor either deep convective or congestus clouds. It also serves to amplify the downdrafts on the boundary layer resulting from detrainment of shallow clouds and evaporation of stratiform rain, which at the same time moisten the upper troposphere. Finally, the deep convective heating is handled in a quasi-equilibrium manner using a BettsMiller-like relaxation scheme. Therefore, the new model combines physical effects presented in both low-level convergence driven and quasi-equilibrium models.
The linear stability analysis about a standard RCE solution performed in sections 3 and 4 revealed scaleselective instability of convectively coupled gravity waves moving at 15 to $20 \mathrm{~m} \mathrm{~s}^{-1}$ at the planetary and synoptic scales. The growth rates and the precise range of instability depend strongly on the strength of the lower tropospheric coupling of the convective parameterization, through the second baroclinic potential temperature, $\theta_{2}$, and the second baroclinic moisture convergence, $\tilde{\lambda}\left(\partial u_{2} / \partial x\right)$, as discussed in section 5 . The heating and fluid dynamical fields of these moist gravity waves are similar to those encountered in the MS01 model that are reminiscent of the moist Kelvin waves as observed, for example, by Wheeler and Kiladis (1999) and Straub and Kiladis (2002), including their eastward propagation speed, the tilt in zonal wind and temperature, the upward motion dominating the heating region, the trailing stratiform part, etc. A notable new feature of the present models, also present in observations, is congestus clouds leading to moistening of the lower troposphere as preconditioning for deep convection.

However, the restriction of the present study to the case without rotation limits the equatorial wave spectrum captured by the model to the convectively coupled Kelvin wave. Although Kelvin waves play a major role in convective coupling, the behavior of other tropical waves is also of interest. Also the use of a uniform Newtonian cooling may seem not suitable for the multicloud parameterization, especially because the present parameterization has an explicit moisture equation and handles three different cloud types, a more adequate radiative scheme should depend upon the moisture distribution and the cloud cover. These issues will be addressed elsewhere by the authors in the near future.

Figure 5 and the budget analysis in section 5 indicate that the basic instability operating in the present models without WISHE is completely different from the stratiform instability in MS01.

The role of the nonlinear switches in the present model on the structure and dynamics of the convectively coupled waves can be fully understood only through nonlinear simulations. This is done in Part II (Khouider and Majda 2005d, manuscript submitted to J. Atmos. Sci.).

Acknowledgments. The research of B.K. is supported by a University of Victoria start-up grant and a grant from the Natural Sciences and Engineering Research Council of Canada. The research of A.M. is partially supported by Grants ONR N0014-96-1-0043, NSF DMS-0456713, and NSF-FRG DMS-0139918. 


\section{APPENDIX A}

\section{Derivation of a Vertically Integrated Moisture Equation for Two-Layer Mode Models}

Recall the vertically averaged equation for the water vapor content, $q$, in the troposphere

$$
\frac{\partial \bar{q}}{\partial t}+\overline{\operatorname{div}(\mathbf{V} q)}+w \frac{\overline{d Q(z)}}{d z}=\frac{1}{H_{T}} F_{-}^{q}-P,
$$

where the overbar represents the vertical averaging and $H_{T}$ is the height of the troposphere (see Frierson et al. 2004 and references therein). Here $\mathbf{V}$ is the horizontal velocity field, $w$ is the vertical velocity, $F_{-}^{q}$ is the moisture flux at the surface, and $P$ is the bulk precipitation rate. Because of the constraint of conservation of moist static energy, the net moisture input; that is, $\left(1 / H_{T}\right) F_{-}^{q}$, is set in (2.2) to be equal to the downdrafts, $\left(1 / H_{T}\right) D$.

When the governing (primitive) equations are Galerkin projected onto the first two baroclinic vertical modes plus a barotropic mode, the velocity field takes the form

$$
\begin{aligned}
& \mathbf{V} \approx \overline{\mathbf{U}}+\mathbf{v}_{1} G(z)+\mathbf{v}_{2} G(2 z) \\
& w \approx-\frac{H_{T}}{\pi}\left(G^{\prime}(z) \operatorname{div} \mathbf{v}_{1}+\frac{1}{2} G^{\prime}(2 z) \operatorname{div} \mathbf{v}_{2}\right),
\end{aligned}
$$

where $\mathbf{v}_{1}$ and $\mathbf{v}_{2}$ are the first and second baroclinic velocity components with $G(z)=(2)^{1 / 2} \cos \left(\pi z / H_{T}\right), G^{\prime}(z)$ $=(2)^{1 / 2} \sin \left(\pi z / H_{T}\right)$ are the associated basis functions, and $\overline{\mathbf{U}}$ is the barotropic component. Plugging the last formulas for $\mathbf{V}$ and $w$ into the equation in (A1) yields approximately

$$
\begin{aligned}
\frac{\partial \bar{q}}{\partial t} & +\overline{\mathbf{U}} \cdot \nabla \bar{q}+\operatorname{div}\left[\mathbf{v}_{1} \phi(q)\right]+\operatorname{div}\left[\mathbf{v}_{2} \psi(q)\right] \\
& +\tilde{Q}\left(\operatorname{div} \mathbf{v}_{1}+\tilde{\lambda} \operatorname{div} \mathbf{v}_{2}\right)=\frac{1}{H_{T}} F_{-}^{q}-P
\end{aligned}
$$

where

$$
\phi(q)=\frac{1}{H_{T}} \int_{0}^{H_{T}} q G(z) d z ; \quad \psi(q)=\frac{1}{H_{T}} \int_{0}^{H_{T}} q G(2 z) d z
$$

and

$$
\begin{gathered}
\tilde{Q}=-\frac{1}{\pi} \int_{0}^{H_{T}} \frac{d Q(z)}{d z} G^{\prime}(z) d z ; \\
\tilde{\lambda} \tilde{Q}=-\frac{1}{2 \pi} \int_{0}^{H_{T}} \frac{d Q(z)}{d z} G^{\prime}(2 z) d z .
\end{gathered}
$$

By assuming plausible vertical profiles for $Q(z)$ and $q$ we derive here some rough estimates for the quantities $\phi(q), \psi(q), \tilde{Q}$, and $\tilde{\lambda}$ in (A.3) and (A.4). We start with $\tilde{Q}$ and $\tilde{\lambda}$. The observed vertical distribution of water vapor in the Tropics (Emanuel 1994; Pruppacher and Klett 2000) suggests a profile for $Q(z)$ that is decreasing rapidly in the lower troposphere and asymptotically vanishing aloft. Here we assume an exponential form profile,

$$
Q(z)=q_{0} \exp \left(-z / H_{q}\right),
$$

where $H_{q}$ is the $e$-folding distance or the moisture-scale height and $q_{0}=Q(z=0)$ is the background surface moisture. We have, from (A.4),

$$
\tilde{Q}=\frac{q_{0}}{\pi H_{q}} \int_{0}^{H_{T}} e^{-z / H_{q}} G^{\prime}(z) d z=\frac{\sqrt{2} q_{0}}{1 / \tau+\pi^{2} \tau}\left(1+e^{-1 / \tau}\right)
$$

and

$$
\begin{aligned}
\tilde{\lambda} \tilde{Q} & =\frac{q_{0}}{2 \pi H_{q}} \int_{0}^{H_{T}} e^{-z / H_{q}} G^{\prime}(2 z) d z \\
& =\frac{\sqrt{2} q_{0}}{1 / \tau+4 \pi^{2} \tau}\left(1-e^{-1 / \tau}\right),
\end{aligned}
$$

where $\tau=H_{q} / H_{T}$. This yields

$$
\tilde{\lambda}=\tilde{\lambda}(\tau)=\frac{1+\pi^{2}(\tau)^{2}}{1+4 \pi^{2}(\tau)^{2}} \tanh \left(\frac{1}{2 \tau}\right)
$$

a monotonically decreasing function of $\tau=H_{q} / H_{T}$ with the upper-bound $\tilde{\lambda} \leq \lim _{\tau \rightarrow 0} \tilde{\lambda}(\tau)=1$. If, for example, $H_{q} / H_{T}=1 / 8$, then $\tilde{\lambda}=0.7135$. Therefore, values of $\tilde{\lambda}$ ranging from $\tilde{\lambda}=0.7$ to $\tilde{\lambda}=1$ are plausible. We use the conservative value of $\tilde{\lambda}=0.8$. Moreover, note that $\tilde{Q}$ depends linearly on the surface moisture $q_{0}$ therefore it can take any arbitrary value. For our calculations we use the standard value of $\tilde{Q}=0.9$ also used in (Emanuel 1987; Yano and Emanuel 1991; Frierson et al. 2004).

For $\phi(q)$ and $\psi(q)$, we seek closures on the form

$$
\phi(q) \approx \delta \bar{q} ; \quad \text { and } \quad \psi(q) \approx \tilde{\delta} \bar{q},
$$

where $\delta$ and $\tilde{\delta}$ are constants. Assuming that the bulk of the moisture variability is concentrated within the lower half of the troposphere, a similar study as for $\tilde{Q}$ and $\tilde{\lambda}$ above, leads to the approximate values of $\delta \approx 1$, $\tilde{\delta} \approx 0.1$. More details about the present derivation are given in Khouider and Majda (2005c).

\section{APPENDIX B}

\section{Linearized Equations about RCE}

For the reader's convenience, we formulate here the linearized equations, about an RCE solution, discussed in section 3. Notice that in the case of a purely deep- 
convective RCE; that is, when $\bar{\theta}_{\mathrm{eb}}-\bar{\theta}_{\mathrm{em}}<\theta^{-}$, the switch parameter $\Lambda$ as given by (2.5) is fixed to its RCE value $\bar{\Lambda}=\Lambda_{*}$ and is insensitive to small perturbations in the prognostic variables, $u_{j}, \theta_{j}, j=1,2, \theta_{\mathrm{eb}}, q, H_{s}, H_{c}$. This is not the case however for the mixed congestus/ deep-convective RCE; that is, when $\theta^{-}<\bar{\theta}_{\mathrm{eb}}-\bar{\theta}_{\mathrm{em}}<$ $\theta^{+}$. Therefore the two different RCEs need to be treated differently and they yield two different linear systems. Let $\Lambda=\bar{\Lambda}+\epsilon \lambda$, where the perturbation $\lambda$ is set to zero in the purely deep convective case and it is a linear function of $\theta_{\mathrm{eb}}, \theta_{1}, \theta_{2}$, and $q$, in the mixed case. The latter presents an additional contribution to the dynamics. More precisely, the linearized equations take the general form

$$
\begin{aligned}
& \frac{\partial u_{j}}{\partial t}-\frac{\partial \theta_{j}}{\partial x}=-c_{d}\left(u_{0}\right) u_{j}-\frac{1}{\tau_{D}} u_{j}, j=1,2 \\
& \frac{\partial \theta_{1}}{\partial t}-\frac{\partial u_{1}}{\partial x}=\frac{\pi}{2 \sqrt{2}} P-\frac{1}{\tau_{R}} \theta_{1} \\
& \frac{\partial \theta_{2}}{\partial t}-\frac{1}{4} \frac{\partial u_{2}}{\partial x}=-\frac{\pi}{2 \sqrt{2}} H_{s}+\frac{\pi}{2 \sqrt{2}} H_{c}-\frac{1}{\tau_{R}} \theta_{2} \\
& \frac{\partial \theta_{\mathrm{eb}}}{\partial t}=-\frac{1}{\tau_{e}} \theta_{\mathrm{eb}}-\frac{D}{h_{b}} \\
& \frac{\partial q}{\partial t}+\tilde{Q}\left(\frac{\partial u_{1}}{\partial x}+\tilde{\lambda} \frac{\partial u_{2}}{\partial x}\right)=-P+\frac{1}{H_{T}} D \\
& \frac{\partial H_{s}}{\partial t}=\frac{1}{\tau_{s}}\left(\alpha_{s} P-H_{s}\right) \\
& \frac{\partial H_{c}}{\partial t}=\frac{1}{\tau_{c}}\left(\alpha_{c} \frac{\bar{\Lambda}-\bar{\Lambda}_{*}}{1-\Lambda_{*}} \frac{D}{H_{T}}-\frac{\alpha_{c} \lambda}{1-\Lambda_{*}} \frac{\bar{D}}{H_{T}}-H_{c}\right),
\end{aligned}
$$

where $P, D$, and $\lambda$ are the linear perturbations for the precipitation, the downdrafts, and the switch parameter, respectively, given by

$$
\begin{aligned}
& P=\frac{1-\bar{\Lambda}}{1-\Lambda_{*}} \frac{1}{\tau_{\mathrm{conv}}}\left[a_{1} \theta_{\mathrm{eb}}+a_{2} q-a_{0}\left(\theta_{1}+\gamma_{2} \theta_{2}\right)\right]-\frac{1}{1-\Lambda_{*}} \bar{P}_{0} \lambda \\
& D=m_{0} \bar{\Lambda}\left\{\left(1+\mu_{2} \frac{Q_{R, 2}^{0}}{\bar{P}}\right)\left[\theta_{\mathrm{eb}}-\frac{2 \sqrt{2}}{\pi}\left(\theta_{1}+\alpha_{2} \theta_{2}\right)-q\right]+\frac{\mu_{2}}{\bar{P}}\left(\bar{\theta}_{\mathrm{eb}}-\bar{\theta}_{\mathrm{em}}\right)\left(H_{s}-H_{c}\right)\right\}+\bar{D}_{0} \lambda \\
& \lambda=\left\{\begin{array}{cc}
0 & \text { if } \bar{\theta}_{\mathrm{eb}}-\bar{\theta}_{\mathrm{em}}<\theta^{-}(\text {Deep-convective RCE }) \\
A\left[\theta_{\mathrm{eb}}-\frac{2 \sqrt{2}}{\pi}\left(\theta_{1}+\alpha_{2} \theta_{2}\right)-q\right] & \text { if } \theta^{-}<\bar{\theta}_{\mathrm{eb}}-\bar{\theta}_{\mathrm{em}}<\theta^{+} \text {(Mixed RCE). }
\end{array}\right.
\end{aligned}
$$

\section{REFERENCES}

Arakawa, A., and W. H. Shubert, 1974: Interaction of a cumulus cloud ensemble with the large-scale environment. Part I. $J$. Atmos. Sci., 31, 674-701.

Biello, J., and A. J. Majda, 2005: A new multiscale model for the Madden-Julian oscillation. J. Atmos. Sci., 62, 1694-1721.

Charney, J. G., and A. Eliassen, 1964: On the growth of the hurricane depression. J. Atmos. Sci., 21, 68-75.

Craig, G. C., and S. L. Gray, 1996: CISK or WISHE as the mechanism for tropical cyclone intensification. J. Atmos. Sci., 53, $3528-3540$.
Dunkerton, T. J., and F. X. Crum, 1995: Eastward propagating 2to 15-day equatorial convection and its relation to the tropical intraseasonal oscillation. J. Geophys. Res., 100, $25781-$ 25790.

Emanuel, K. A., 1987: An air-sea interaction model of intraseasonal oscillations in the Tropics. J. Atmos. Sci., 44, 2324-2340.

—, 1994: Atmospheric Convection. Oxford University Press, $592 \mathrm{pp}$.

— J. D. Neelin, and C. S. Bretherton, 1994: On large-scale circulations in convecting atmosphere. Quart. J. Roy. Meteor. Soc., 120, 1111-1143. 
Frierson, D., A. J. Majda, and O. Pauluis, 2004: Dynamics of precipitation fronts in the tropical atmosphere. Comm. Math. Sci., 2, 591-626.

Fuchs, Z., and D. Raymond, 2002: Large-scale modes of a nonrotating atmosphere with water vapor and cloud-radiation feedbacks. J. Atmos. Sci., 59, 1669-1679.

Gill, A. E., 1982: Atmosphere-Ocean Dynamics. Academic Press, $666 \mathrm{pp}$.

Haertl, P. T., and G. N. Kiladis, 2004: On the dynamics of two-day equatorial disturbances. J. Atmos. Sci., 61, 2707-2721.

Hayashi, Y., 1971: Large-scale equatorial waves destabilized by convective heating in the presence of surface friction. $J$. Meteor. Soc. Japan, 49, 458-466.

Johnson, R. H., T. M. Rickenbach, S. A. Rutledge, P. E. Ciesielski, and W. H. Schubert, 1999: Trimodal characteristics of tropical convection. J. Climate, 12, 2397-2407.

Khouider, B., and A. J. Majda, 2005a: A non-oscillatory well balanced scheme for an idealized tropical climate model. Part I: Algorithm and validation. Theor. Comp. Fluid Dyn., 19, 331354.

_ , and 2005b: A non-oscillatory well balanced scheme for an idealized tropical climate model. Part II: Nonlinear coupling and moisture effects. Theor. Comp. Fluid Dyn., 19, 355375 .

$\longrightarrow$, and —, 2005c: Multicloud convective parametrizations with crude vertical structure. Theor. Comp. Fluid Dyn., in press.

Kiladis, G. N., K. Straub, and P. Haertl, 2005: Zonal and vertical structure of the Madden-Julian oscillation. J. Atmos. Sci., 62, 2790-2809.

Lin, X., and R. H. Johnson, 1996: Kinematic and thermodynamic characteristics of the flow over the western Pacific warm pool during TOGA COARE. J. Atmos. Sci., 53, 695-715.

Lindzen, R. S., 1974: Wave-CISK in the Tropics. J. Atmos. Sci., 31, 156-179.

Majda, A. J., and M. Shefter, 2001a: Waves and instabilities for model tropical convective parameterizations. J. Atmos. Sci., 58, 896-914.

$\longrightarrow$, and $-2001 \mathrm{~b}$ : Models for stratiform instability and convectively coupled waves. J. Atmos. Sci., 58, 1567-1584.

— for tropical convection. Proc. Nat. Acad. Sci. USA, 99, 11231128.

— and baroclinic Rossby waves. J. Atmos. Sci., 60, 1809-1821.

—, and —, 2004: A multi-scale model for the intraseasonal oscillation. Proc. Nat. Acad. Sci. USA, 101, 4736-4741.

—, B. Khouider, G. N. Kiladis, K. H. Straub, and M. G. Shefter,
2004: A model for convectively coupled tropical waves: Nonlinearity, rotation, and comparison with observations. J. At mos. Sci., 61, 2188-2205.

Mapes, B. E., 1993: Gregarious tropical convection. J. Atmos. Sci., 50, 2026-2037.

, 2000: Convective inhibition, subgridscale triggering energy, and "stratiform instability" in a toy tropical wave model. $J$. Atmos. Sci., 57, 1515-1535.

Moncrieff, M. W., and E. Klinker, 1997: Organized convective systems in the tropical western Pacific as a process in general circulation models: A TOGA-COARE case study. Quart. J. Roy. Meteor. Soc., 123, 805-827.

Neelin, J. D., and J. Yu, 1994: Modes of tropical variability under convective adjustment and Madden-Julian oscillation. Part I: Analytical theory. J. Atmos. Sci., 51, 1876-1894.

- and N. Zeng, 2000: A quasi-equilibrium tropical circulation model-Formulation. J. Atmos. Sci., 57, 1741-1766.

Pruppacher, H. R., and J. D. Klett, 2000: Microphysics of Clouds and Precipitation. Kluwer, $954 \mathrm{pp}$.

Slingo, J. M., and Coauthors, 1996: Intraseasonal oscillation in 15 atmospheric general circulation models: Results from an AMIP diagnostic subproject. Climate Dyn., 12, 325-357.

Straub, K. H., and G. N. Kiladis, 2002: Observations of a convectively coupled Kelvin wave in the eastern Pacific ITCZ. $J$. Atmos. Sci., 59, 30-53.

Wheeler, M., and G. N. Kiladis, 1999: Convectively coupled equatorial waves: Analysis of clouds and temperature in the wavenumber-frequency domain. J. Atmos. Sci., 56, 374-399.

$\_, \ldots$, and P. J. Webster, 2000: Large-scale dynamical fields associated with convectively coupled equatorial waves. J. Atmos. Sci., 57, 613-640.

Yamasaki, M., 1969: Large-scale disturbances in a conditionally unstable atmosphere in low latitudes. Pap. Meteor. Geophys., 20, 289-336.

Yano, J.-I., and K. A. Emanuel, 1991: An improved model of the equatorial troposphere and its coupling to the stratosphere. $J$. Atmos. Sci., 48, 377-389.

— J. C. McWilliams, M. W. Moncrieff, and K. A. Emanuel, 1995: Hierarchical tropical cloud systems in an analog shallow-water model. J. Atmos. Sci., 52, 1723-1742.

_ , M. W. Moncrieff, and J. C. McWilliams, 1998: Linear stability and single column analyses of several cumulus parametrization categories in a shallow-water model. Quart. J. Roy. Meteor. Soc., 124, 983-1005.

Zehnder, J. A., 2001: A comparison of convergence- and surfaceflux-based convective parameterizations with applications to tropical cyclogenesis. J. Atmos. Sci., 58, 283-301. 